1866 , and again in 1867 , these patients suffering from enlarged spleen associated with leucocythrmia, and both sank almost immediately after the operation.

Since Mr. Bryant's second case in 1867 , splenotomy has been performed in different paits of the world iu no less than twenty.two cases of diseased conditions of the gland, and some twenty-six times for injuries implicating the spleen. Dr. Nussbaum, on whose authority I make the latter statement, ${ }^{3}$ inform 3 us that sixteen out of these twenty-six operations proved quite successful. I will dismiss this class of cases with the above notice, and make a few remarks on the subject of splenotomy bing a justifiable operation in leucocythæmia.

It will be seen from the Table that out of a total of twenty-nine cases splenotomy has been performed no less than sixteen times for leucocythæmia, and on thirteen occasions for various other morbid conditions of the spleen. It is equally apparent that out of the thirteen cases in which leucocythæmia was absent, no less than eight recoveries are recorded; while out of the sixteen in which the latter disease was present there is no instance in which the patient recovered even from the primary effects of the operation. Now, it must strike the most casual observer that the fatality, in these latter cases, is due to something outside the mere effect of the operation, which in itself appears to be less dangerous than one might reasonably be led to expect. And, surely, few will deny, although many appear to forget, that this "something" is actually present in these cases, and readily explains the cause of surgeons losing at the rate of a hundred per cent. after splenotomy in leucocythæmia.

Let me here quote from the pen of Di. Gowers, in Reynolds' "System of Medicine," ${ }^{ \pm}$who, $a$ propos this subject, remarks: "In leucocythæmia the tendenev to hæmorrhage is unquestionably greater than in most case s of simple splenic anæmia, and this constitutes a danger so great as to render the operation [splenotomy] scarcely justifiable in the advanced stages of the disease. Naturally a risk of immediate death so great as is and must be involved in the pperation is only consented to when the disease is advanced, and the odema and dyspncea in themselves indicative of the greatest source of risk -the profound blood change. We are yet, moreover, without any evidence as to whether the removal of the spleen would be attended with an improvement in the deficiency of the red globules, which constitutes the greatest source of danger." Dr. Gowers tells us there is no evidence to prove that removal of the spleen would produce any alteration in the relation of white and red globules; and although it must be admitted that we possess no such evidence from cases in which the operation has been performed for leucocythæmiafor the simple reason the it no case has lived to tell the tale-we do possess valuable evidence to prove that in some of those cases which have recovered after extirpation of the spleen for simrile disease, the normal relation between the two sets of corp.uscles has remained unchanged. ${ }^{\breve{b}}$ In fact, what little we kniow of the pathology of leucocythæmia is certainlv in e all the lymphatic structures of the body, as well as the blood stream, and but little likely to be influenced by the removal of any one part concerned. Professor Mosler, ${ }^{6}$ writing on excision of the spleen as a remedy in leucocythæmia, thus concludes his remarks : "These facts lead us to the conclusion that neither in the early nor in the late stages of leucocythremia is splenotomy justifiable; and, indeed, that operations even of the most trifling character are to be as far as possible avoided."

I will close this paper by quoting Mr. Bryant's concluding remarks upon the result of his two cases. ${ }^{7} \mathrm{He}$ writes: "We have thus learnt two things from the cases related. Firstly, that the enlargement of the spleen in leucocythænia appears to be only a part of a general disease affecting the glandular system as a whole; and, secondly, that in splenotomy, for such a disease there is a predisposition to hæemorrhage, with which surgery is incompetent to deal. It can neither be foreseen by any amount of care, nor coped with by any amount of skill. Under these circumstances there is no shirking the conclusion that the operation is physiologically unsound and surgically unsafe, and for leucocythæmia should not be performed." To these

2 Vide Guy's Hospital Reports, Series iii., vols. xii., xiii., pp. 444,411 3 Vide Nussbaum's Deutsche Chirurgie. 4 Vol. v., page 304. 5 Vide Moulin's case in table, and Brit. Med. Jour., Feb. 9th, 1878.

Vide Guy's Hospital Reports, rol. xiii., page 411 remarks, coming as they do from one who has fully realised upon how slight a fabric the theory of splenotomy as a remedy for leucocythremia is built, it will surely be unnecessary to add more. And, in conclusion, let me trust that the recorded facts, as represented in the appended Table, together with the condemnation of the operation by the high authorities I have quoted, will suffice to prevent the further repetition of this useless and deadly experiment. Turnham Green, $W$

\section{INFLAMMATORY ENLARGEMENT OF BONE.}

BY CHARTERS J. SYMONDS, M.S., F.R.C.S., ASSISTANT-SURGEON AND SURGICAL REGISTRAR TO GUY'S HOSPITAL.

IN Professor Lister's address on the "Relation of Micro. organisms to Inflammation," published in THE LANCET of Oct. 22nd, 1881, there occurs towards the end, a statement which I venture to think is somewhat erroncous. After giving several examples of the influence of nerve action in originating and maintaining inflammation, he relates a case of "white swelling, a gelatinous degeneration of the synovial membrane of the knee-joint," in which it is said that "sympathetically the bones also had become affected, and the ends of both the tibia and the femur were greatly enlarged." This remark from one so prominent must necessarily carry with it the weight of authority, and in attempting to show that it is at least difficult to conceive such a condition occurring in a bone from simple ostitis, I am fully aware of our respective positions in the field of pathological research.

As the subject of hypertrophy of bone is one of consider. able breadth, I would first state that the object of the present communication is an attempt to show that all in. flammatory enlargement in bone is due to periosteal deposit; and that simple caries never increases the size of a bone until it invades the periosteum, when the latter, where healthy, lays down a variable amount of new material around the carious area. It seems a matter of indifference as regards enlargement, whether the ostitis be a primary one, or, as in Professor Lister's case, secondary to synovial disease; nor does it seem to matter whether the inflammatory product suppurate, caseate, or organise.

The term "sympathetic hypertrophy," as applied to bone, appears to indicate some connexion with the increase of secreting glands, such as the mamma, and to be either tem. porary or permanent. Temporary swelling of a bone, from a vaso-motor condition, will, I imagine, be allowed, from the nature of things, to be impossible. Even admitting a permanent increase from central changes, which I hope to show later on may be otherwise explained, it is difficult to imagine what becomes of the articular cartilage, itself a non-vascular structure intimately connected with the bone.

Having, during the past two years, as Surgical Registrar of Guy's Hospital, had frequent opportunities of examining the excised articular ends of bones, particularly of the femur and tibia, I have carefully investigated the question of enlargement, with the result that in no case has this change been observed in pulpy disease. The joints have exhibited all stages of gelatinous degeneration, the bones in some being extensively carious. In other specimens removed by amputation, no enlargement was found even with advanced necrosis of the articular surfaces. Those cases where stalactitic-like masses surround the articular ends-a condition allied apparently to the changes in rheumatic goutare of course not included, the increase here being a surface one, and occurring only in the middle-aged.

In the clinical examination of these cases there always appears to be enlargement of the articular ends, more especially of the lower extremity of the femur. This, however, is explained, first, by the fact that the extreme wasting of the thigh throws out into unnatural prominence the femoral condyles. By pressing up the structures behind the bone, and so bringing the transverse diameter of the thigh nearly equal to the sound side, the undue prominence to a great extent disappears. There is, however, a distinct increase in the size of the joint, varying in the transverse measure. ment from one-eighth to one-fourth of an inch, an amount easily explained by the thickening of the synovial membrane and ligaments from infiltration of the granulation 
tissue; for when unrestrained, this material, as in the suprapatellar pouch, may measure an inch and a half in thickness.

As the above may seem but a contradiction and an opinion, though substantiated by numerous specimens both in $\mathrm{my}$ own possession and in the museum at this hospital, it is possible to show that the same holds true in other parts of a bone. For example, in central ostitis resulting in suppuration, as in the extremities of the tibia, it is well known that there is at first no enlargement; and if the inflamed area be small, the pus may remain for a long period enclosed within the bone, without any increase in size taking place. When, however, the inflammatory process has reached the periosteum, then, and I would say only then, is there any enlargement.

The example of enlargement usually explained by chronic ostitis is that where on section the bone is found uniformly cancellous to the outer compact layer. Such a bone is said to be "expanded" by an enlargement of the normal spaces, or to be "swollen." Thus Sir Jas. Paget, ${ }^{1}$ after describing the process of inflammatory softening in bone, proceeds to show the subsequent changes that are permitted, "especially the swelling and expansion." In the first specimen used to illustrate the subject, that of a humerus removed from an arm four months after excision of the elbow, he says, "The lower end was red and swollen, with expansion or separation of the layers of its walls; and the case showed well the coincidence of absorption and of enlargement by expansion.' In describing the next illustration, which exhibits an in crease in diameter, due to the presence of regular lamina outside a compact layer, Sir James says, "The most striking change is a more or less extensive and wide separation of the concentric laminæe of the walls of the bone, so that, as in the section of this femur (Fig. 46), the longitudinal section of the enlarged wall appears composed of two or more layers of compact lissue with a widely cancellous tissue between them, and these layers may sometimes be traced into con. tinuity with those forming the healthy portion of the wall." In a previous paragraph he describes bone as " yielding and extending as the naturally softer tissues do in an inflamma tory swelling."

I venture, in opposition to this explanation, to submit the following, founded upon the observation that all enlargement seems, from the examination of specimens, to be periosteal. The uniformity on section is explained in two ways. In some specimens, as in Sir James Paget's first case, I would suggest that there is periostitis at the same time as the ostitis. That the former process lays down new bone and increases the diameter, while the latter leads to absorption of the bony framework, and to widening of the cancellous spaces and of the smaller cavities in the compact layer. The new bone being itself cancellous, and the compact having become so, there is an unbroken area of this material from the medullary canal to the thin compact layer bounding the new periosteal bone. The result is that expansion appears to have taken place. When the inflammatory products have organised and the bone becomes sclerosed, and when the dense periosteal layer is blended with the original compact tissue, the latter can still be traced by its greater whiteness, and usually greater density, an additional proof of the periosteal increase. In the same way it seems to me that the narrowing of the medullary canal may be explained by the formation of new bone, by the endosteum, and not, as Sir James Paget says, by ostitis alone. On thi point he writes, "The inner layers of the wall are pressed inwards, and encroach upon the medullary tissue."

In other cases the periostitis is more chronic, and the ostitis superficial only, so that more or less of the old compact layer remains after all inflammation has subsided. Such a specimen, I fancy, is Sir James Paget's second illus tration above referred to (Fig. 46). In such I would suggest the following interpretation. New bone is deposited by periostitis, the associated ostitis is superficial, and hence there is little cancellation of the compact layer from this cause If the periosteal bone become permaneat, with or without passing through the sclerotic stage, then the original compact layer becomes cancellous. This seems to be effected by a physiological process independent of inflammation. The compact layer now occupies the interior of the bone, it no longer has the function of compact tissue, and so slowly assumes the character of the surrounding material. This may actually be seen taking place, as in a specimen in the

1 Lectures on Surgical Pathology, 4th edit., pp. 297,298 .
Guy's Museum (No. 1153), in which the sclerosed periosteal bone has become blended with the compact layer. The cancellating process has in one part removed all distinction between the two. It seems, in fact, to follow from the functions of the several parts of a bone that, given a permanent increase in diameter from periosteal deposit, the surface of that deposit will become compact and blend above and below with the original compact laver of the bone; while the deeper part, though having become sclerosed, will, with the now embedded compact layer, ultimately become porous, and the two be indistinguishable from each other and from the original cancellous tissue.

In support of this view, it may be mentioned that the impacted compact layer in fractures becomes cancellous, suggesting that all parts within the outer compact layer must in a healthy condition of bone assume this condition. ${ }^{2}$

Smith also, in his work on Fractures (p. 161), in endeavouring to disprove the existence of impaction in Colles* fracture, says :- "At a still more remote period the enveloped portion of compact structure is frequently removed by absorption ; it becomes, as it were, resolved into cancellated structure, the appearance of penetration is effaced, and the whole interior of the bone presents a cellular aspect.'

This functional modelling in new bone is seen in the union of fractures with overlapping of the fragments. Here the periosteal formation is the bond of connexion, and where it passes from the end of one fragment to the surface of the other it has an outer compact and an inner cancellous part.

The following statements embody the views above explained :-

1. That ostitis, even when central, is only attended with enlargement when the periosteum is involved.

2. That in all cases of primary synovial disease of joints, or of caries of articular surfaces, with secondary synovial disease, there is no enlargement of the bone.

3. That the enlargement of joint-ends in these cases is only apparent, and is explained by the unnatural prominence of the femoral condyles, owing to wasting of the thigh and to the thickening of the ligaments and synovial membrane.

4. That all inflammatory increase in the size of bones is due to periosteal formation, and that the uniformly cancellated appearance is due $(a)$ to the widening from absorption of the spaces in the compact layer, whereby it becomes indistinguishable from the original cancellous tissue and the porous periosteal bone; and $(b)$ to the remodelling of the compact layer when the ostitis has been superficial.

5. That the term "expansion" is misleading as applied to the results of the inflammatory processes in bone, since the widening of the spaces is an absorptive one.

St. Thomas's-street, S.E.

\section{ON THE GRANULAR MATTER OF THE BLOOD. ${ }^{1}$}

BY E. H. HOWLETT, F.R.C.S.,

RESIDENT SURGICAL OFFICER, MANCHESTER ROYAL INFIRMARY.

THE blood may shortly be described as consisting of a fluid or plasma, and a solid or corpuscular portion. The latter is composed chiefly of two varieties: (1) the white corpuscles or leucocytes ; (2) the red corpuscles or erythrocytes (Alex. Duane). In addition, there is also a considerable amount of granular matter; and it is to some of these granules that my attention has recently been directed. If a drop of blood be smeared on a slide, rapidly dried, stained, and mounted, after the manner proposed by Koch, as a ready method of examining fluids for micro-organisms, it will be found that certain portions only are stained-viz, the nuclei of the leucocytes, whilst the body as a rule remains clear; sometimes the whole corpuscle becomes stained. The red discs do not take up the colouring matter unless the staining is excessive, and then only to a slight degree. It is somewhat surprising at first how, in a

An impacted fracture of humerus, No. $1111(50)$ shows this.

1 In justice to the author we feel it desirable to state that the MS. of this article came into our hands in the middle of November last year prior, therefore, to the publication of Bizzozero's research 3s, commented on in our issue of Jan, 21st.-ED. L. 